\title{
Evaluación de la cosecha de plantas seleccionadas de henequén (Agave fourcroydes Lem) y propagadas in vitro
}

\author{
Gerardo González (Ing. Agrónomo PhD) \\ Silvia Alemán (Ing. en Agrómo MSc.) \\ Hilter Figueroa Saavedra (Ing. Agrónomo MSc.) \\ Arisdorgan Diéguez (Lic. en Matemáticas MSc.) \\ Ramiro Herrera (Ing. Administración y Producción \\ Agropecuaria MSc) \\ Universidad de Matanzas. Autopista a Varadero, Matanzas, Cuba \\ Universidad / Escuela Superior Politécnica de Chimborazo Extensión Norte \\ Amazónica de la República del Ecuador
}

Doi: 10.19044/esj.2018.v14n33p17 URL:http://dx.doi.org/10.19044/esj.2018.v14n33p17

\begin{abstract}
This paper focuses on the establishment of a system of in vitro cloning of henequen, of which the behavior of these plants in production conditions is unknown. With the objective of evaluating the agricultural yields in selected henequen and plants propagated in vitro, five harvests were carried out during the years 2005 to 2009. In the five harvest, the yields in fiber (tn/ha) were higher in the vitroplantas in regards to conventionally propagated plants. The first harvest was done three years after the plantation was carried out. In all crops, line 4 statistically surpassed lines 1 and 9 in terms of the fibre content, which showed a greater productive potential. The histological characterization of these plants showed a greater number of vascular bundle and strands of fibers in the vitroplants with regard to plants multiplied by the conventional method. This, however, showed a significant correlation with more fibre content in the first.
\end{abstract}

Keywords: Henequen, In vitro propagation, selection

\section{Resumen}

Se estableció un sistema de clonación in vitro del henequén, del cual no se conoce su comportamiento en condiciones de producción. Con el objetivo de evaluar los rendimientos agrícolas en plantas seleccionadas de henequén y propagadas in vitro, se realizaron cinco cosechas durante los años 
2005 a 2009. En las cinco cosechas, los rendimientos en fibra (t/ha) fueron superiores en las vitroplantas con relación a plantas propagadas convencionalmente. Se produjo la primera cosecha a los tres años de realizada la plantación. En todas las cosechas, la línea 4 superó estadísticamente a las líneas 1 y 9 en cuanto al contenido de fibra, lo que evidenció un mayor potencial productivo. La caracterización histológica mostró un mayor número de haces conductores y cordones de fibras en las vitroplantas, con respecto a las plantas multiplicadas por el método convencional, lo que se correlaciona con más contenido de fibras en las primeras.

Palabras Claves: Henequén, propagación in vitro, selección

\section{Introducción}

El imperativo de la economía mundial a favor de la conservación de los recursos bióticos y su diversidad genética, y de la disminución de la contaminación por plásticos y otros productos dañinos al hombre y al ambiente, precisa de la caracterización, recuperación y conservación de los recursos genéticos disponibles para hacer de ellos un uso más racional (Garriga et al., 2006).

En la actualidad, la tendencia al uso de métodos de explotación sustentables, así como las exigencias de disminuir la contaminación del medio por productos no degradables, unido al incremento del precio internacional de la fibra, ha elevado la demanda mundial de ésta (González et al., 2007). Todo ello ha despertado un interés muy marcado en Cuba, donde se le está brindando una mayor atención a este cultivo (Abreu et al., 2007), de origen yucateco, el cual fue introducido en el país a mediados del pasado siglo. Existe, además, el interés de que con este cultivo se contribuya a la eliminación de importaciones, las cuales afectan la economía nacional por su incremento.

La evaluación de la cosecha de las vitroplantas de henequén permite conocer las potencialidades de este tipo de posturas y facilita la introducción de este tipo de material en la producción. Sin lugar a dudas, las vitroplantas constituyen un complemento a las posturas de baja calidad que se extraen de las plantaciones actuales y son un nuevo método de obtención de plantas como una vía alternativa de propagación que se espera contribuya, en menor tiempo, a que se logren plantaciones de Henequén homogéneas y de alta calidad en el país.

\section{Metodología}

\section{Evaluación de caracteres morfológicos}

A los dos años de sembradas las vitroplantas, en condiciones de plantación comercial, se evaluaron 50 plantas de cada línea. Para el análisis 
se evaluaron: longitud de la hoja $(\mathrm{cm})$, ancho de la hoja en el medio $(\mathrm{cm})$, ancho de la hoja en la base $(\mathrm{cm})$, número de espinas, distancia entre espina (cm) y masa fresca $(\mathrm{g})$.Con baseen los valores obtenidos, se realizó un análisis de componentes principales.

\section{Evaluación del comportamiento en la producción de fibras de líneas propagadas in vitro}

Las vitroplantas fueron cosechadas teniendo en cuenta la separación de éstas en tres líneas (L-1, L-4 y L-9), siguiendo criterios establecidos por González et al. (2003).

Durante cinco años se evaluó la producción de fibras en las tres líneas propagadas in vitro", comparadas con plantas propagadas por la vía convencional.

\section{Análisis histológico}

Los tejidos de la lámina fueron seccionados en pequeños trozos de 2 mm, obtenidos de la zona media de la lámina de la hoja y colocados en el fijador (Paraformaldehído al $4 \%$ ). Estos tejidos se sometieron a condiciones de vacío de 3 a 5 minutos para ayudar a la penetración del fijador dentro de la muestra. Posteriormente, se mantuvieron con el fijador durante 18 a 24 horas a una temperatura de $4{ }^{\circ} \mathrm{C}$; después se lavaron las muestras en tampón PBS (Phosphate Buffered Saline) y se le realizaron tres cambios de 10 minutos cada uno.

Una vez fijadas las muestras, se procedió a deshidratarlas en etanol y, finalmente, fueron incluidas en resina Epón. Los cortes semifinos de $1 \mu \mathrm{m}$ de espesor se tiñeron con azul de toluidina y se montaron en euquit. Fueron fotografiados con una cámara digital Olympus, acoplada al microscopio.

\section{Resultados y Discusión}

\section{Evaluación de caracteres morfológicos}

Al realizar un análisis de componentes principales (Figura 1), a 150 individuos propagados in vitro (50 por planta donante) y sus tres plantas donantes, se observó que los dos primeros componentes explicaron el 94,3\% de la variación total del ensayo de campo. Las variables asociadas al rendimiento en fibra: ancho de la hoja en la región basal (V3) y masa fresca de la hoja (V6), más la variable no deseada: distancia entre espinas (V5), tuvieron la mayor contribución al componente C1 $(79,3 \%)$ y la variable asociada al rendimiento: longitud de la hoja al componente C2 (15\%).

A partir de estos resultados, dado que la propagación vegetativa del henequén se realiza fundamentalmente a través de hijos de rizoma (Robert et al., 1992), se evidencia la existencia de variaciones genéticas en individuos 
seleccionados, a partir de caracteres morfológicos pertenecientes a una población, por lo que sugiere la posibilidad de seleccionar individuos.

Figura 1. Distribución de los individuos micropropagados y sus donantes en los planos 1 y 2 del análisis de componentes principales.

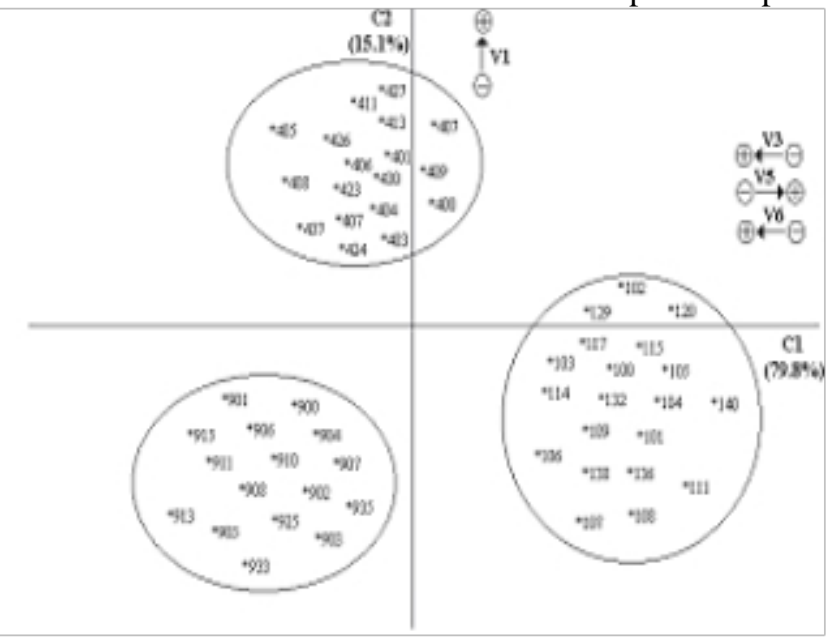

\begin{tabular}{crr}
\hline \multicolumn{3}{c}{ Vectores propios } \\
\hline & $\mathrm{C} 1$ & \multicolumn{1}{c}{$\mathrm{C} 2$} \\
\hline V1- & 0,19 & 0,93 \\
V2- & $-0,42$ & 0,22 \\
V3- & $-0,44$ & 0,10 \\
V4- & $-0,42$ & 0,21 \\
V5- & 0,44 & $-0,01$ \\
V6- & $-0,44$ & 0,12 \\
\hline
\end{tabular}

C1, C2: Dos primeros componentes principales.

Al: Individuos Línea 1 (100 al 150).

A2: Individuos Línea 4 (400 al 450).

A3: Individuos Línea 9 (900 al 950).

V1: Longitud de la hoja.

V3: Ancho de la hoja en la base.

V5: Distancia entre espina.

V6: Masa fresca de la hoja.

La Tabla 1 muestra el comportamiento del desarrollo y de la cosecha de las líneas propagadas. Las plantas producidas in vitro fueron más homogéneas en todos sus aspectos morfológicos y fisiológicos que las plantas generadas en campo, lo que contribuyó a que las primeras lograran una sincronización de crecimiento tal que casi más del 90 \% de éstas llegaron a la etapa de su ciclo de producción al mismo tiempo. Contrario sucedió con las segundas, donde menos del $40 \%$ llegó a esta etapa.

Tabla 1. Resultados de la cosecha de las líneas propagadas in vitro y de plantas propagadas por la vía tradicional después de tres años de plantación definitiva.

\begin{tabular}{|c|c|c|c|c|c|}
\hline Tp & 1 & 2 & 3 & 4 & 5 \\
\hline L-1 & $0,6 \mathrm{~b}$ & $0,7 \mathrm{~b}$ & $0,9 \mathrm{~b}$ & $0,9 \mathrm{~b}$ & $0,9 \mathrm{~b}$ \\
L-4 & $0,8 \mathrm{a}$ & $0,9 \mathrm{a}$ & $1,2 \mathrm{a}$ & $1,3 \mathrm{a}$ & $1,3 \mathrm{a}$ \\
L-9 & $0,7 \mathrm{~b}$ & $0,7 \mathrm{~b}$ & $0,9 \mathrm{~b}$ & $0,9 \mathrm{~b}$ & $1,0 \mathrm{~b}$ \\
PC & $0,2 \mathrm{c}$ & $0,4 \mathrm{c}$ & $0,6 \mathrm{c}$ & $0,6 \mathrm{c}$ & $0,7 \mathrm{c}$ \\
Es & 0,1 & 0,2 & 0,1 & 0,2 & 0,2 \\
\hline
\end{tabular}

Medias con letras diferentes representan diferencias significativas entre las variantes $(\mathrm{p} \leq$ $0,05)$. 
Tp: Tipo de planta; del 1 al 5 representan las cosechas de los años 2005, 2006, 2007, 2008 y 2009, respectivamente.

$L-1$; $L-4$; $L-9$ : representan las líneas propagadas "in vitro".

$P C$ : representa las plantas propagadas por vía convencional.

Es: Error estándar.

Este resultado evidencia la superioridad de las vitroplantas, pues debido a su mayor velocidad de crecimiento alcanzan la altura mínima requerida para la cosecha de las hojas a los tres años y cuatro meses, en vez de a los cinco o seis años que requieren las plantas propagadas por la vía tradicional.

Los resultados de cosecha indican la superioridad en producción de fibras por parte de las vitroplantas en comparación con las plantas producidas por la vía tradicional. En las cinco cosechas se muestra que las primeras superaron estadísticamente en rendimiento en fibra a las segundas. Dentro de las vitroplantas, la línea 4 superó significativamente en t/ha de fibra a las líneas 1 y 9 en todas las cosechas; sin embargo, entre estas dos últimas líneas no se produjeron diferencias estadísticas.

Las figuras 2 y 3 muestran la diferencia en el número de haces conductores entre las vitroplantas (L-4) y las plantas propagadas tradicionalmente. Las primeras superaron en cantidad de haces conductores a las segundas. Este resultado histológico corroboró por qué las vitroplantas presentaron mayor rendimiento en fibra, pues es alrededor de los haces conductores que se producen los paquetes de fibras.

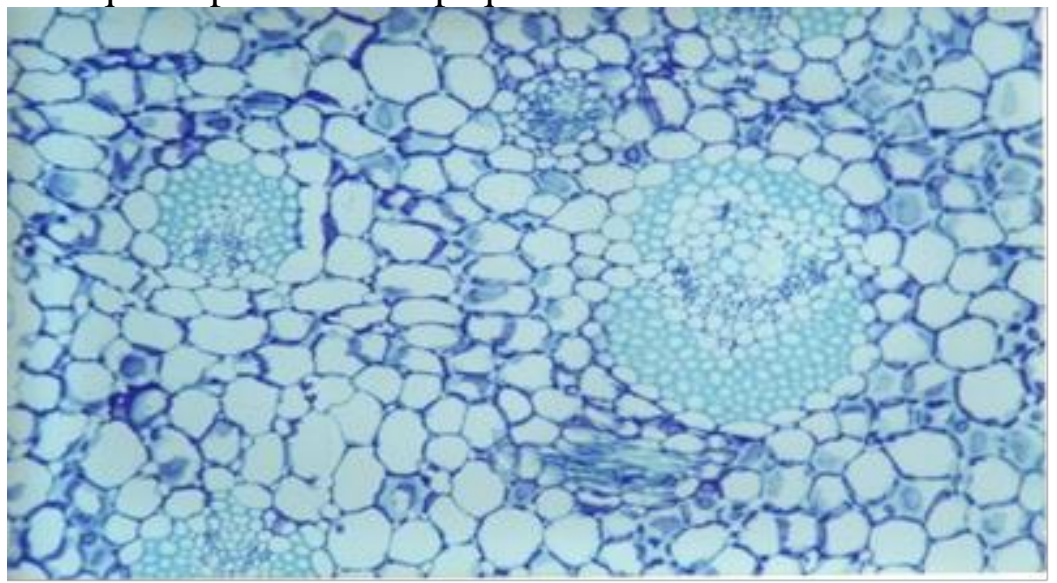

Figura 2. Distribución de los haces conductores y cordones de fibra en vitroplanta (L-4). Las flechas lo indican.

Se conoce que uno de los efectos de las auxinas se caracteriza por estimular la presencia de cambios morfológicos como el incremento de la proliferación del tejido vascular. 
Según Aloni et al. (2006), la disminución del nivel de transcripción de ATHB15 (factor de transcripción de expresión exclusiva en tejidos vasculares) ocasiona diferenciación celular vascular acelerada a partir de células cambiales/procambiales, fenómeno que pudiera ser el responsable del incremento del número de haces conductores en las vitroplantas y con ello el incremento del rendimiento en fibras.

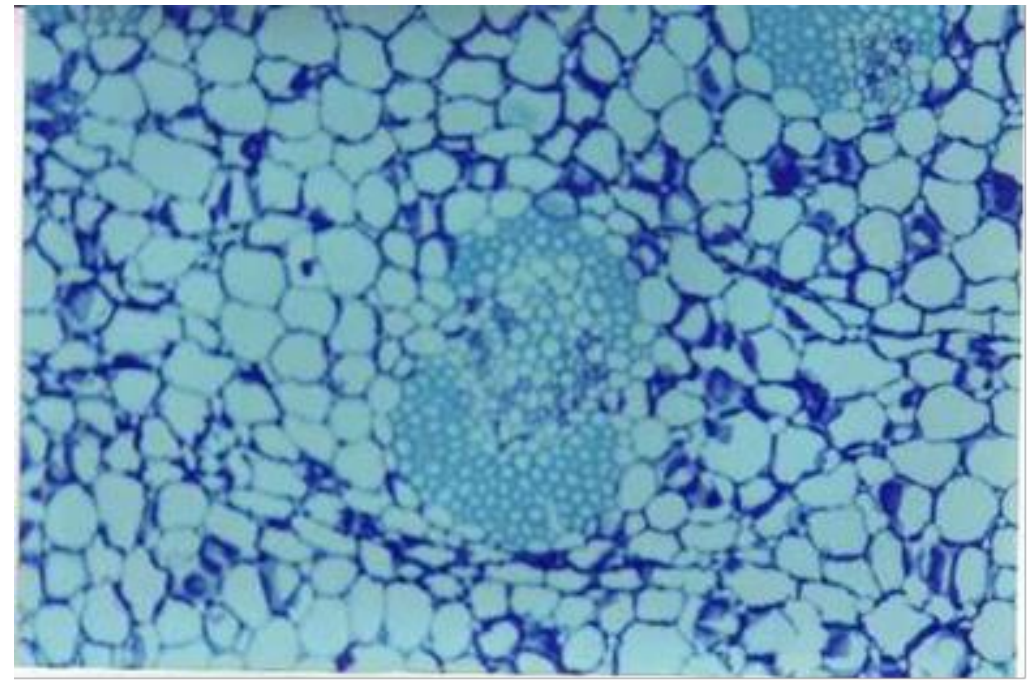

Figura 3. Distribución de los haces conductores y cordones de fibra en plantas de campo.

Las flechas lo indican.

Este resultado evidenció, en primer lugar, que la presencia de auxinas en los medios de cultivo pudiera favorecer la diferenciación de los haces conductores $\mathrm{y}$, en segundo lugar, la rejuvenilización incrementa el metabolismo celular, lo que conlleva a que las plantas generen una mayor circulación de sustancias y, por lo tanto, sugiere un mayor número de haces conductores.

\section{Conclusiones}

La selección y el cultivo in vitro favorecieron el incremento en fibra de las vitroplantas, y estas producen mayor número de fibra con relación a las plantas propagadas convencionalmente, de modo que la combinación de la selección en campo y la clonación in vitro puede convertirse en una vía sostenible para la recuperación henequenera.

\section{References:}

1. Banco Mundial (2017). Banco Mundial. [En línea]. Available at: http://datos.bancomundial.org/indicador/SL.UEM.TOTL.ZS [Último acceso: 2811 2017]. 
2. CONEVAL (2017). CONEVAL. [En línea]. Available at: http://www.coneval.org.mx/Medicion/Paginas/Cohesion_Social.aspx [Último acceso: 2911 2017].

3. Cortés, M. M. S. J. P. P. S. F. (2018). Biblioteca Virtual Clacso. [En línea]. Available at: http://bibliotecavirtual.clacso.org.ar/ar/libros/costar/flacso/cuad131.p df

4. INIDE (2016). Instituto Nacional deInformación de Desarrollo. [En línea]. Available at: http://www.inide.gob.ni/Emnv/Emnv14/EMNV\%2020142\%20Febrero\%202016.pdf [Último acceso: 3011 2016].

5. Ortiz, L. P. (2009). Universidad Autónoma de Madrid. [En línea]. Available at: https://www.uam.es/personal_pdi/economicas/laurap/EEM/3Desigualdad.pdf [Último acceso: 201611 29].

6. Pampillón, R. (2009). Economy weblog. [En línea]. Available at: http://economy.blogs.ie.edu/archives/2009/10/\%C2\%BFque-es-elindice-de-desarrollo-humano-idh.php [Último acceso: 2811 2016].

7. Peralta, E. (2010). Perspectiva Laboral en México. comercio exterior, pp. 195-208.

8. Sen Amartya, K. (1992). Inequality Reexamined. Cambridge: Harvard University, 01 01, pp. -

9. Torres, A. (2017). Debate. [En línea]. Available at: https://www.debate.com.mx/mexico/La-desigualdad-tiene-su-ladopositivo-Deaton-20170922-0276.html 